PROCEEDINGS OF THE

AMERICAN MATHEMATICAL SOCIETY

Volume 131, Number 6, Pages 1953-1964

S 0002-9939(02)06800-4

Article electronically published on October 24, 2002

\title{
DEFORMATIONS OF MINIMAL LAGRANGIAN SUBMANIFOLDS WITH BOUNDARY
}

\author{
ADRIAN BUTSCHER
}

(Communicated by Bennett Chow)

\begin{abstract}
Let $L$ be a special Lagrangian submanifold of a compact CalabiYau manifold $M$ with boundary lying on the symplectic, codimension 2 submanifold $W$. It is shown how deformations of $L$ which keep the boundary of $L$ confined to $W$ can be described by an elliptic boundary value problem, and two results about minimal Lagrangian submanifolds with boundary are derived using this fact. The first is that the space of minimal Lagrangian submanifolds near $L$ with boundary on $W$ is found to be finite dimensional and is parametrized over the space of harmonic 1-forms of $L$ satisfying Neumann boundary conditions. The second is that if $W^{\prime}$ is a symplectic, codimension 2 submanifold sufficiently near $W$, then, under suitable conditions, there exists a minimal Lagrangian submanifold $L^{\prime}$ near $L$ with boundary on $W^{\prime}$.
\end{abstract}

\section{Introduction AND StATEMENT OF RESUlts}

A minimal Lagrangian submanifold of a symplectic manifold $M$ is at once minimal with respect to the metric of $M$ and Lagrangian with respect to the symplectic structure of $M$. Furthermore, when $M$ is a Calabi-Yau manifold, Harvey and Lawson showed in their seminal paper [3, Section III] that minimal Lagrangian submanifolds are also calibrated. A consequence of this property is that minimal Lagrangian submanifolds satisfy a relatively simple geometric PDE: $L \subset M$ is minimal Lagrangian if and only if

$$
\begin{gathered}
\left.\operatorname{Im}\left(\mathrm{e}^{\mathrm{i} \theta} \Omega\right)\right|_{L}=0, \\
\left.\omega\right|_{L}=0,
\end{gathered}
$$

for some real number $\theta$. Here, $\omega$ is the symplectic form of $M$ and $\Omega$ is the canonical, non-vanishing, holomorphic $(n, 0)$-form of $M$, whose existence is guaranteed by the Calabi-Yau structure of $M$.

The calibration form defined on $M$ is in this case $\boldsymbol{R e}\left(\mathrm{e}^{\mathrm{i} \theta} \Omega\right)$ and thus $\left.\boldsymbol{R e}\left(\mathrm{e}^{\mathrm{i} \theta} \Omega\right)\right|_{L}$ $=\mathrm{Vol}_{L}$. The submanifold $L$ is also referred to as special Lagrangian with phase angle $\theta$ in the literature, and if $L$ is minimal Lagrangian with phase angle $\theta=0$, then $L$ is simply called special Lagrangian.

Harvey and Lawson have exploited the geometric structure implicit in the calibration condition in order to tackle questions related to the existence of minimal Lagrangian submanifolds. Harvey and Lawson themselves produce several examples of minimal Lagrangian submanifolds and give certain general constructions

Received by the editors October 11, 2001 and, in revised form, January 24, 2002.

2000 Mathematics Subject Classification. Primary 58J05. 
of such objects. More recently, Schoen and Wolfson [16] have presented another construction based on variational methods and are investigating the singularities that can arise there, while Haskins [4] has constructed new examples of special Lagrangian submanifolds and cones. Many further advances have been made recently by Joyce, for example in [6], [7, 8], [9], [10, 11], [12.

Another approach for producing minimal Lagrangian submanifolds involves studying the deformations of a given minimal Lagrangian candidate $L$ and selecting those deformations of $L$ which preserve the minimal Lagrangian condition. A deformation of a submanifold $L \subset M$ is a continuous family of embeddings $f_{t}: L \rightarrow M$ of $L$, where $f_{0}$ is the canonical embedding. The goal of this analysis is to characterize the space of submanifolds near $L$ which are still minimal and Lagrangian. This will be carried out by applying perturbative techniques to the equations satisfied by minimal Lagrangian submanifolds.

The first results in this area were obtained by McLean [14, Section 3] and extended by Hitchin [5]. Using (10), McLean identified the first order deformations of a special Lagrangian submanifold in a Calabi-Yau manifold and developed a method for integrating them. He used this to characterize the space of special Lagrangian submanifolds near $L$ according to the following theorem.

Theorem (McLean, 1996). Let $M$ be a compact, Calabi-Yau manifold. The space of special Lagrangian submanifolds sufficiently near a given candidate $L \subset M$ is finite dimensional and is parametrized over the set $\mathcal{H}^{1}(L)$ of harmonic one-forms of $L$.

McLean's work has been extended to the case where $M$ is symplectic by Salur [15]. The work presented in this paper extends McLean's result in another direction - this time to minimal Lagrangian submanifolds with non-empty boundary in a Calabi-Yau manifold $M$. This will be done by first creating a framework for incorporating boundary conditions into the minimal Lagrangian differential equations. More precisely, the boundary conditions will arise through geometric restrictions on the deformations of the special Lagrangian submanifolds. An object that will be called a scaffold will be used for this purpose.

Definition 1. Let $L$ be a submanifold of $M$ with boundary $\partial L$ and inward unit normal vector field $N \in \Gamma\left(T_{\partial L} L\right)$. A scaffold for $L$ is a smooth submanifold $W$ of $M$ with the following properties:

(1) $\partial L \subset W$;

(2) $N \in \Gamma\left(T_{\partial L} W\right)^{\omega}$ (here, $S^{\omega}$ denotes the symplectic orthogonal complement of a subspace $S$ of a symplectic vector space $V$, defined as $S^{\omega} \equiv\{v \in V$ : $\omega(v, s)=0 \forall s \in S\})$;

(3) the bundle $(T W)^{\omega}$ is trivial.

Remarks. Condition (2) is a transversality condition that ensures that $J N$ is perpendicular to $W$, where $J$ is the complex structure of $M$. It seems reasonable to expect the Main Theorem to hold with condition (2) replaced by an unconstrained transversality condition, but this weaker assumption leads to technical problems later on. In particular, the boundary value problem appearing in the analysis of the linearized operator in Section 3.2 is the Hodge system with oblique boundary conditions rather than the Hodge system with Neumann boundary conditions. Since this BVP is more difficult to deal with and leads to geometrically less natural results, the author has avoided it here. Furthermore, it is possible that the most 
geometrically natural type of scaffold is when $W$ is a complex submanifold of $M$ (i.e. the tangent spaces of $W$ are invariant under $J$ ) and this automatically satisfies the transversality condition (2).

Further remarks. Condition (3) will be used in the sequel in order to make certain constructions on $W$ possible; also, the above definition of a scaffold has already been used in [2].

The boundary condition on the deformation is imposed in the following way. Suppose that, under deformation, $\partial L$ is confined to move only along the scaffold $W$. In other words, if $f_{t}: L \longrightarrow M$ is a deformation, then suppose that $f_{t}(\partial L) \subset W$ for all $t$. Consequently, the deformation field $V=\left.\frac{\mathrm{d}}{\mathrm{d} t} f_{t}\right|_{t=0}$ cannot be arbitrary: it must be tangent to $W$ at $\partial L$. These considerations lead to the statement of the Main Theorem to be proved in this paper.

Main Theorem (Boundary Deformation Theorem). Let $L$ be a special Lagrangian submanifold of a compact Calabi-Yau manifold $M$ with non-empty boundary $\partial L$ and let $W$ be a symplectic, codimension two scaffold for $L$. Then the space of minimal Lagrangian submanifolds sufficiently near $L$ (in a suitable $C^{1, \beta}$ sense to be defined later on) but with boundary on $W$ is finite dimensional and is parametrized over the harmonic 1-forms of $L$ satisfying Neumann boundary conditions.

The remainder of this paper will be organized in the following manner. In Section 2 the boundary value problem describing minimal Lagrangian submanifolds with boundary on a scaffold is formulated, and in Section 3 the proof of the main theorem is undertaken by solving this boundary value problem. The Implicit Function Theorem is to be used for this purpose, so the linearized operator corresponding to the BVP must be calculated and shown to be surjective with finite dimensional kernel isomorphic in a suitable sense to the harmonic 1-forms of $L$. At the end of this paper, the Boundary Deformation Theorem will be used to prove a corollary, which is an existence result for minimal Lagrangian submanifolds with boundary in $M$.

\section{Formulating the Boundary Value Problem}

2.1. Introduction. For the remainder of this paper, assume that $L$ is a given, fixed special Lagrangian submanifold with boundary that is contained in an ambient $2 n$-real-dimensional Calabi-Yau manifold $M$, and that $M$ possesses a metric $g$, a symplectic form $\omega$, and compatible complex structure $J$. Denote by $\Omega$ the canonical, holomorophic, non-vanishing $(n, 0)$-form of $M$. Furthermore, assume that $L$ is connected; the results for non-connected $L$ follow simply by considering each component of $L$ separately. The equations (1) satisfied by minimal Lagrangian submanifolds suggest the definition of a map whose zero set corresponds to the minimal Lagrangian submanifolds near $L$. Let $\operatorname{Emb}(L, M)$ denote the set of embeddings of $L$ into $M$ (we will worry about regularity later) and denote by $\Lambda^{k}(L)$ the $k$-forms of $L$. Now define $\Phi: \operatorname{Emb}(L, M) \times \mathbf{R} \rightarrow \Lambda^{1}(L) \times \Lambda^{n}(L)$ by

$$
\Phi(f, \theta)=\left(f^{*} \omega, f^{*} \operatorname{Im}\left(\mathrm{e}^{\mathrm{i} \theta} \Omega\right)\right) .
$$

Since $L$ itself is special Lagrangian, $\Phi\left(i_{L}, 0\right)=(0,0)$, where $i_{L}$ is the canonical embedding of $L$. Another minimal Lagrangian embedding of $L$, with calibration angle $\theta$, is an embedding $f$ satisfying $\Phi(f, \theta)=(0,0)$. 
The Main Theorem of this paper consists of finding those embeddings of $L$ near $i_{L}$ which satisfy $\Phi(f, \theta)=(0,0)$ for some $\theta$ by means of the Implicit Function Theorem. The precise version of this theorem that will be employed is the following.

Theorem 2 (Implicit Function Theorem). Let $F: B \rightarrow Z$ be a smooth map of Banach spaces with $F(0)=0$. Suppose that there exist closed Banach subspaces $X$ and $Y$ of $\mathcal{B}$ so that $\mathcal{B}=X \oplus Y$. If $\mathrm{D}_{X} F(0)$ is bijective, then there is a neighbourhood $\mathcal{U}$ of 0 in $Y$ and a $C^{1}$ map $\phi: \mathcal{U} \rightarrow X$ so that $\phi(0)=0$ and $F(y+\phi(y))=0$ for all $y \in \mathcal{U}$.

See [1, Section 2.5] for an excellent discussion of this theorem as well as its proof. The Implicit Function Theorem thus provides families of solutions of the equation $F(b)=0$, parametrized over the Banach subspace which complements the subspace on which the linearization of $F$ at 0 is bijective. Note that in the case where $\mathrm{D} F(0)$ is surjective with finite dimensional kernel $K$, the Implicit Function Theorem holds with $Y=K$ and $X$ equal to any Banach subspace, necessarily closed, that complements $K$. The Main Theorem will be proved using this special case, while the corollary will be proved using the more general statement.

The map $\Phi$, as defined earlier, does not yet involve Banach spaces. Thus in order to apply the Implicit Function Theorem to $\Phi$, a sufficiently large class of embeddings of $L$ near $i_{L}$ must be parametrized over a Banach space, and the equation $\Phi(f, \theta)=(0,0)$ must be solved in this Banach space.

2.2. Imposing boundary conditions with a scaffold. In order to understand why boundary conditions must be imposed on the deformations of $L$, one must consider the linearization of the operator $\Phi$ at the point $\left(i_{L}, 0\right)$.

Proposition 3. Let $\Phi: \operatorname{Emb}(L, M) \times \mathbf{R} \rightarrow \Lambda^{1}(L) \times \Lambda^{n}(L)$ be the operator defined in (2). The linearization of $\Phi$ at the point $\left(i_{L}, 0\right)$ is given by

$$
\mathrm{D} \Phi\left(i_{L}, 0\right)(V, a)=\left(\mathrm{d} \eta, \mathrm{d} \star \eta+a \operatorname{Vol}_{L}\right),
$$

where $V$ is a vector field defined on $L$, a is a real number and $\left.\eta=i_{L}^{*}(V\rfloor \omega\right)$.

Proof. Let $f_{t}: L \rightarrow M$ be a family of embeddings with $f_{0}=i_{L}$ and $\left.\frac{\mathrm{d}}{\mathrm{d} t} f_{t}\right|_{t=0}=V$, and let $a_{t}$ be a family of real numbers with $a_{0}=0$ and $\left.\frac{\mathrm{d}}{\mathrm{d} t} a_{t}\right|_{t=0}=a$. Now,

$$
\mathrm{D} \Phi\left(i_{L}, 0\right)(V, a)=\left.\frac{\mathrm{d}}{\mathrm{d} t} \Phi\left(f_{t}, a_{t}\right)\right|_{t=0} .
$$

The calculation of the derivative of $\Phi$ in the $f_{t}$ direction has already been carried out by McLean in [14]. It remains only to perform the calculation in the $a_{t}$ direction. This can be done by differentiating

$$
\begin{aligned}
\left.\frac{\mathrm{d}}{\mathrm{d} t} \Phi(0, t a)\right|_{t=0} & =\left.\left(0,-\frac{\mathrm{d}}{\mathrm{d} t} \mathbf{I m}\left(\mathrm{e}^{-\mathrm{i} t a} \Omega\right)\right)\right|_{t=0} \\
& =\left(0, a i_{L}^{*}(\mathbf{R e}(\Omega))\right) \\
& =\left(0, a \mathrm{Vol}_{L}\right)
\end{aligned}
$$

by definition of a calibration form. This calculation, in combination with McLean's result, completes the proof of the proposition.

Boundary conditions are necessary because the Hodge operator $\eta \mapsto(\mathrm{d} \eta, \mathrm{d} \star \eta)$ is not elliptic unless it acts upon a space of differential 1-forms satisfying certain 
boundary conditions. From the Hodge theory on manifolds with boundary [18, it is known that one such boundary condition is the Neumann boundary condition, which is when forms $\eta$ satisfy $\eta(N)=0$ along $\partial L$, where $N$ is the unit normal vector field of $\partial L$ in $L$. In the case under consideration here, $\eta$ arises as the 1-form associated to a deformation of a special Lagrangian submanifold, and is thus of the form $\eta=V\rfloor \omega$, where $V=\left.\frac{\mathrm{d}}{\mathrm{d} t} f_{t}\right|_{t=0}$ is the corresponding deformation vector field. The Neumann boundary condition thus translates into the condition $\omega(V, N)=0$. The following proposition shows that this boundary condition arises naturally if the deformations of $L$ force the boundary of $L$ to remain on a scaffold as in the statement of the Main Theorem.

Proposition 4. Let $L$ be a special Lagrangian submanifold of $M$ and let $W$ be a symplectic, codimension two scaffold for $L$. Let $f_{t}: L \longrightarrow M$ be any deformation of $L$ satisfying $f_{t}(\partial L) \in W$ for all $t$. Then the deformation vector field $V=\left.\frac{\mathrm{d}}{\mathrm{d} t} f_{t}\right|_{t=0}$ corresponding to $f_{t}$ satisfies the Neumann boundary condition.

Proof. The vector field $V$ must be parallel to $W$ along $\partial L$ as indicated in the Introduction. But according to the transversality component of the definition of a scaffold, $N \in\left(T_{x} W\right)^{\omega}$ for every $x \in \partial L$. Therefore $\omega(N, V)=0$.

2.3. Constructing scaffold preserving deformations. In the proof of McLean's Theorem, deformations of $L$ are parametrized over the Banach space of $C^{1, \beta}$ sections of the normal bundle of $L$ using the exponential map. That is, for every section $V$ of the normal bundle of $L$, the exponential map defines an embedding of $L$ via $\exp (V): L \rightarrow M$. Exponential deformations are, however, not suitable for the present purpose, because in general $\exp (V)(\partial L)$ will not lie on $W$ because $W$ is in general not totally geodesic. Another means of deforming $L$ is thus necessary if $\partial L$ is to remain confined to the scaffold under deformation. One way to avoid the difficulty described above is to consider the exponential map of a different metric $\hat{g}$ - one in which $W$ is totally geodesic.

Before the metric $\hat{g}$ can be constructed, a lemma concerning the local structure of $W$ near $\partial L$ is needed. This is essentially a version of the Lagrangian Neighbourhood Theorem [13, page 99] that is valid for Lagrangian submanifolds with boundary.

Lemma 5. Let $W$ be a symplectic submanifold of codimension 2 in $M$ and suppose that $L$ is a Lagrangian submanifold with boundary $\partial L \subset W$. Then there exists a tubular neighbourhood $\mathcal{U}$ of the boundary and a symplectomorphism $\psi: \mathcal{U} \longrightarrow$ $T^{*}(\partial L \times \mathbf{R})=T^{*}(\partial L) \times \mathbf{R}^{2}$ with the following properties:

(1) $\psi(W \cap \mathcal{U}) \subset T^{*}(\partial L) \times\{0,0\}$;

(2) $\psi(\partial L)=\partial L \times\{0,0\}$;

(3) $\psi(L \cap \mathcal{U}) \subset \partial L \times \mathbf{R}_{+} \times\{0\}$; and

(4) let $E$ be any non-zero section of $(T W)^{\omega}$ and denote by $\left(s^{1}, s^{2}\right)$ the coordinates of the $\mathbf{R}^{2}$ factor. Then $\psi$ can be constructed so that $\psi_{*}(E)=\frac{\partial}{\partial s^{1}}$.

Proof. Because $W$ is symplectic, the symplectic form $\left.\omega\right|_{W}$ makes $W$ a symplectic manifold in its own right. Since $\partial L$ is an isotropic submanifold of $M$ with respect to $\omega$, it is a compact Lagrangian submanifold of $W$ with respect to $\left.\omega\right|_{W}$. Consequently, the usual Lagrangian Neighbourhood Theorem can be applied to $\partial L$ as a submanifold of $W$ to produce a neighbourhood $\mathcal{U}_{0}$ and a symplectomorphism $\psi_{0}: \mathcal{U}_{0} \longrightarrow T^{*}(\partial L)$. The desired symplectomorphism $\psi$ will be found by extending $\psi_{0}$ off $W$ in a suitable way. 
The Symplectic Neighbourhood Theorem [13, page 98] will be used to complete the extension. The theorem applies to two symplectic manifolds $\left(M_{1}, \omega_{1}\right)$ and $\left(M_{2}, \omega_{2}\right)$ containing symplectic submanifolds $W_{1}$ and $W_{2}$, respectively. It states that if there exists a symplectic vector bundle isomorphism $\Psi:\left(T W_{1}\right)^{\omega} \longrightarrow$ $\left(T W_{2}\right)^{\omega}$ that covers a symplectomorphism $\psi: W_{1} \longrightarrow W_{2}$, then there exist neighbourhoods $\mathcal{U}_{1}$ and $\mathcal{U}_{2}$ of $W_{1}$ and $W_{2}$ respectively, along with a symplectomorphism $\psi_{e}: \mathcal{U}_{1} \longrightarrow \mathcal{U}_{2}$ that extends $\psi$ (that is, $\left.\psi_{e}\right|_{W_{1}}=\psi$ ).

Let $M_{1}=M, W_{1}=W, M_{2}=T^{*}(\partial L) \times \mathbf{R}^{2}$ and $W_{2}=T^{*}(\partial L) \times\{0,0\}$. Let $s^{1}$ and $s^{2}$ be the coordinate functions in the $\mathbf{R}^{2}$ factor. One of the defining conditions for a scaffold implies that its sympectic normal bundle $(T W)^{\omega}$ is trivial. Hence it is possible to choose two vector fields $E$ and $F$ which span $(T W)^{\omega}$ and satisfy $\omega(E, F)=1$. Extend this basis to the neighbourhood $\mathcal{U}_{0}$ and continue to denote the extended vector fields by $E$ and $F$. Define an isomorphism $\Psi:(T W)^{\omega} \longrightarrow \mathbf{R}^{2}$ of symplectic vector bundles by

$$
\Psi\left(E_{x}\right)={\frac{\partial}{\partial s^{1}}}_{\left(\psi_{0}(x), 0,0\right)} \quad \text { and } \quad \Psi\left(F_{x}\right)=\frac{\partial}{\partial s^{2}}\left(\psi_{0}(x), 0,0\right)
$$

at any $x \in \mathcal{U}_{0}$. This clearly covers the symplectomorphism $\psi_{0}$ and is a symplectic map.

The Symplectic Neighbourhood Theorem can now be invoked to yield a symplectomorphism $\psi_{1}$ extending $\psi_{0}$ between some tubular neighbourhood of $\partial L$ and a neighbourhood of $\partial L \times\{0,0\}$ in $T^{*}(\partial L) \times \mathbf{R}^{2}$. Only the third requirement on the symplectomorphism is not met by $\psi_{1}$. However, by composing with a suitable symplectomorphism that acts as a translation in the transverse Lagrangian directions to $L$, this condition can also be achieved.

The adapted coordinates for a neighbourhood of $\partial L$ of the preceding lemma, with $E=N$, can now be used to construct the metric $\hat{g}$. This is done in three separate steps.

Step 1 . Let $\mathcal{U}$ be the tubular neighbourhood of $\partial L$ provided by Lemma 5 and $\psi: \mathcal{U} \longrightarrow T^{*}(\partial L) \times \mathbf{R}^{2}$ the symplectomorphism, with $\psi_{*}\left(\frac{\partial}{\partial s^{1}}\right)=N$. Now define the metric $g_{1}$ at the point $\left(x, y, s^{1}, s^{2}\right) \in T^{*}(\partial L) \times \mathbf{R}^{2}$ as follows:

$$
g_{1}\left(x, y, s^{1}, s^{2}\right)=\left(\psi^{-1}\right)^{*}\left(\left.g\right|_{W}(\psi(x, y, 0,0))\right)+\mathrm{d} s^{1} \otimes \mathrm{d} s^{1}+\mathrm{d} s^{2} \otimes \mathrm{d} s^{2} .
$$

Step 2. Without loss of generality, the form (5a) can be taken for an entire tubular neighbourhood $\mathcal{U}_{1}$ of $W$. This is because the topological assumption made on $W$ - that $W$ has trivial normal bundle - is enough to guarantee the extension of the coordinates $s^{1}$ and $s^{2}$ to the entire tubular neighbourhood.

Step 3 . Let $\eta: M \longrightarrow \mathbf{R}$ be a positive, $C^{\infty}$ cut-off function which equals 1 inside a tubular neighbourhood $\mathcal{U}_{1}^{\prime}$ of $\partial L$ contained in $\mathcal{U}_{1}$, and equals 0 outside $\mathcal{U}_{1}$. Now define the metric $\hat{g}$ by

$$
\hat{g}=\eta g_{1}+(1-\eta) g .
$$

It remains to verify that the metric $\hat{g}$ brings about the desired properties. These are confirmed by the following two propositions.

Proposition 6. The submanifold $W$ is totally geodesic with respect to the metric $\hat{g}$ constructed in (5). 
Proof. Let $\frac{\partial}{\partial z^{1}}, \ldots, \frac{\partial}{\partial z^{2 n-2}}$ be a set of local coordinate vector fields for the relatively open neighbourhood $W \cap \mathcal{U}_{1}^{\prime}$. Then $\frac{\partial}{\partial z^{1}}, \ldots, \frac{\partial}{\partial z^{2 n-2}}, \frac{\partial}{\partial s^{1}}, \frac{\partial}{\partial s^{2}}$ determines a set of local coordinate vector fields for the neighbourhood $\mathcal{U}_{1}^{\prime}$. In these coordinates,

$$
\hat{g}=\left(\begin{array}{cc}
\left(\left.g\right|_{W}(z)\right)_{i j} & 0 \\
0 & \delta_{i j}
\end{array}\right) .
$$

Now, $\left\langle\nabla_{\frac{\partial}{\partial z^{2}}} \frac{\partial}{\partial z^{j}}, \frac{\partial}{\partial s^{k}}\right\rangle=\frac{1}{2}\left(\hat{g}_{z^{i} s^{k}, z^{j}}+\hat{g}_{z^{j} s^{k}, z^{i}}-\hat{g}_{z^{i} z^{j}, s^{k}}\right)=0$. This implies that the second fundamental form of $W$ with respect to $\hat{g}$ vanishes; thus $W$ is totally geodesic.

Proposition 7. Let $L$ be a special Lagrangian submanifold with boundary on the symplectic, codimension two scaffold $W$ and let $N$ be the unit normal vector field of $\partial L$ in $L$. Construct the metric $\hat{g}$ according to (5). Suppose $V$ is a section of $\hat{N} L$ that satisfies the boundary condition $\omega(V, N)=0$. Then $V$ is tangent to $W$ over $\partial L$.

Proof. Choose a point $x$ in $\partial L$ and coordinates at $x$ as in the constructions above. Furthermore, assume that $T_{x} \partial L$ is spanned by $\frac{\partial}{\partial z^{1}}, \ldots, \frac{\partial}{\partial z^{n-1}}$ and that $\frac{\partial}{\partial z^{n}}, \ldots$, $\frac{\partial}{\partial z^{2 n-2}}$ are orthogonal to these vectors. Since $N$ equals $\frac{\partial}{\partial s^{1}}$ in these coordinates, it is now easy to see that the $\hat{g}$-normal bundle of $L$ at $x$ is spanned by the vectors

$$
\frac{\partial}{\partial z^{n}}, \ldots, \frac{\partial}{\partial z^{2 n-2}} \text { and } \frac{\partial}{\partial s^{2}}+\lambda \frac{\partial}{\partial s^{1}}
$$

for some $\lambda \in \mathbf{R}$. So, if $V \in \hat{N}_{x} L$ and $\left(\sum \mathrm{d} z^{i} \wedge \mathrm{d} z^{n-1+i}+\mathrm{d} s^{1} \wedge \mathrm{d} s^{2}\right)(V, N)=0$, then clearly the $\frac{\partial}{\partial s^{2}}+\lambda \frac{\partial}{\partial s^{1}}$ component of $V$ must vanish and as a result, $V \in T_{x} W$.

From elementary metric geometry, it is known that $\widehat{\exp }$ is a local diffeomorphism on $\hat{N} L$. Thus the conclusion to be drawn from Proposition [6 and Proposition 7 is that sufficiently small $\hat{g}$-exponential deformations of sections of $\hat{N} L$ satisfying the boundary condition imposed by the scaffold $W$ are in one-to-one correspondence with submanifolds near $L$ with boundary on $W$ that project onto $L$ via $\hat{g}$-nearest point projection.

\section{Proof of the Main Theorem}

3.1. Defining the differential operator. The apparatus created in the previous section for deforming the special Lagrangian submanifold $L \subset M$ such that its boundary remains confined to the scaffold $W$ can now be used to set up a differential equation whose solutions correspond to minimal Lagrangian submanifolds near $L$ with boundary on $W$. Construct the metric $\hat{g}$ and the $\hat{g}$-normal bundle $\hat{N} L$ of $L$ as in the previous section, let $N$ denote the unit normal vector field of $\partial L$, and define the Banach space

$$
\mathcal{X}=\left\{V \in C^{1, \beta}(\Gamma(\hat{N} L)): \omega(V, N)=0\right\}
$$

of vector fields satisfying the Neumann boundary condition imposed by $W$. The notation used here is the following. If $B$ denotes any bundle over $L$, then $\Gamma(B)$ denotes the sections of $B$, and $C^{k, \beta}(\Gamma(B))$ denotes the set of sections whose $k$ covariant derivatives exist and are bounded in the $C^{k, \beta}$ norm, which is given by $|u|_{C^{k, \beta}}=\sum_{i=0}^{k}\left\|\nabla^{i} u\right\|_{0}+\left[\nabla^{k} u\right]_{\beta}$ for any section $u \in \Gamma(B)$, where $\|X\|_{0}$ is the supremum norm of a section $X$ over $L$ and $[X]_{\beta}$ is its Hölder coefficient. 
Next, denote by $\mathrm{d} \Lambda^{k}(L)$ the set of exact $(k+1)$-forms of $L$ and define the operator

$$
\Phi: \mathcal{X} \times \mathbf{R} \rightarrow C^{0, \beta}\left(\mathrm{d} \Lambda^{1}(L)\right) \times C^{0, \beta}\left(\mathrm{d} \Lambda^{n-1}(L)\right)
$$

by

$$
\Phi(V, \theta)=(\widehat{\exp }(V))^{*}\left(\omega,-\mathbf{I m}\left(\mathrm{e}^{-\mathrm{i} \theta} \Omega\right)\right)
$$

where $\widehat{\exp }$ is the $\hat{g}$-exponential map as defined in the previous section. Note that elements of $C^{0, \beta}\left(\mathrm{d} \Lambda^{k}(L)\right)$ are necessarily of the form $\mathrm{d} \eta$ for some $\eta \in C^{1, \beta}\left(\Lambda^{k-1}(L)\right)$ by the Poincaré Lemma and the basic properties of Hölder spaces.

Note. The range of $\Phi$ is indeed the set of exact 1 - and $n$-forms. This is because any element of the range is homotopic to the zero 1 - and $n$-forms, which is obviously exact, and exactness is preserved under homotopy.

3.2. Analysis of the linearized operator. In order to apply the Implicit Function Theorem to the map $\Phi$ in the vicinity of the point $(0,0)$, it is necessary to show that $\Phi$ is a smooth map of Banach spaces and the linearization $\operatorname{D} \Phi(0,0)$ is bounded and surjective, and whose kernel is isomorphic to the finite dimensional set of harmonic 1-forms of $L$ that satisfy Neumann boundary conditions.

The smoothness of $\Phi$ as a Banach space map is straightforward. Recall now the expression of the linearization of the minimal Lagrangian equations from Proposition 3. since $\left.\frac{\mathrm{d}}{\mathrm{d} t} \widehat{\exp }(t V)\right|_{t=0}=V$,

$$
\mathrm{D} \Phi(0,0)(V, a)=\left(\mathrm{d} \eta, \mathrm{d} \star \eta+a \mathrm{Vol}_{L}\right),
$$

where $\left.\eta=i_{L}^{*}(V\rfloor \omega\right)$ as before. This is clearly a bounded operator. Surjectivity is verified in the following theorem.

Proposition 8. The operator $\mathrm{D} \Phi(0,0): \mathcal{X} \times \mathbf{R} \rightarrow C^{0, \beta}\left(\mathrm{d} \Lambda^{1}(L)\right) \times C^{0, \beta}\left(\mathrm{d} \Lambda^{n-1}(L)\right)$ is surjective.

Proof. Let $N$ be the unit normal vector field of $\partial L$ and let $\alpha \in C^{1, \beta}\left(\Lambda^{1}(L)\right)$ and $\beta \in C^{1, \beta}\left(\Lambda^{n-1}(L)\right)$. Consider the system of equations $\mathrm{D} \Phi(0,0)(V, a)=(\mathrm{d} \alpha, \mathrm{d} \beta)$ in the space $\mathcal{X} \times \mathbf{R}$; or in other words, consider

$$
\begin{gathered}
\mathrm{d} \eta=\mathrm{d} \alpha, \\
\mathrm{d} \star \eta=\mathrm{d} \beta+a \mathrm{Vol}_{L}, \\
\eta(N)=0 .
\end{gathered}
$$

Hodge theory for a manifold $L$ with boundary shows that a $k$-form satisfying the equations

$$
\begin{gathered}
\mathrm{d} \eta=\sigma, \\
\mathrm{d} \star \eta=\tau, \\
\eta(N)=0,
\end{gathered}
$$

and possessing a given degree of Hölder regularity can be found if and only if the following conditions are met:

(1) $\mathrm{d} \sigma=0$ and $\mathrm{d} \tau=0$;

(2) $\left.\tau\left(E_{1}, \ldots, E_{k+1}\right)\right|_{\partial L}=0$ for any collection of vectors $E_{i}$ tangent to $\partial L$;

(3) $\int_{L} \sigma \wedge \star \lambda=0$ for every harmonic $(k+1)$-form $\lambda$ of $L$ satisfying Neumann boundary conditions;

(4) $\int_{L} \star \tau \wedge \star \kappa=0$ for every harmonic $(k-1)$-form $\kappa$ of $L$ satisfying Neumann boundary conditions. 
This list of conditions is given in [18, page 123]. Note that these results are actually only stated for $k$-forms with Sobolev regularity. But they extend fairly easily to Hölder regularity by standard techniques of elliptic theory (as explained in [17], for example).

Because of form of the (7), only condition (4) above imposes any restriction on the solvability of these equations. Thus a 1 -form $\eta$ that solves this system of equations can be found if and only if the integrability condition

$$
\int_{L} \mathrm{~d} \beta+a \int_{L} \mathrm{Vol}_{L}=0
$$

can be made to hold. But since $\int_{L} \operatorname{Vol}_{L}=\operatorname{Vol}(L) \neq 0$, one can choose $a=-\frac{\int_{L} \mathrm{~d} \beta}{\operatorname{Vol}(L)}$ to meet the integrability condition.

It remains to find the kernel of the linearized operator in order to complete the proof of the Main Theorem. Suppose that the equations

$$
\begin{gathered}
\mathrm{d} \eta=0, \\
\mathrm{~d} \star \eta+a \mathrm{Vol}_{L}=0, \\
\eta(N)=0,
\end{gathered}
$$

are satisfied by a 1-form $\eta$ on $L$ and a real number $a$. Integrating the second equation over $L$ yields:

$$
\begin{aligned}
\operatorname{a\operatorname {Vol}(L)} & =-\int_{L} \mathrm{~d} \star \eta \\
& =-\int_{\partial L} \star(\eta(N)) \\
& =0
\end{aligned}
$$

where $i_{\partial L}$ is the standard embedding of $\partial L$ in $M$. The calculations above hold by Stokes' Theorem as well as by the properties of the Hodge star operator at the boundary of $L$ (these properties are derived in [18, Sections 1.2 and 2.1]). Hence $a=0$ and $\eta$ satisfies the Hodge system $\mathrm{d} \eta=\delta \eta=0$ with the boundary condition $\eta(N)=0$. The solutions of these equations are the harmonic 1-forms with Neumann boundary conditions. This is a finite dimensional space of dimension equal to $b^{1}(L)$, as found in [18, Section 2.6].

All of the hypotheses required by the Implicit Function Theorem are thus satisfied by the map $\Phi: \mathcal{X} \times \mathbf{R} \rightarrow C^{0, \beta}\left(\mathrm{d} \Lambda^{1}(L)\right) \times C^{0, \beta}\left(\mathrm{d} \Lambda^{n-1}(L)\right)$. Thus if

$$
K=\{V \in \mathcal{X}: \mathrm{D} \Phi(0,0)(V, 0)=(0,0)\}
$$

is the finite dimensional kernel of $\mathrm{D} \Phi(0,0)$, there is a $C^{1}$ map $f: \mathcal{U} \rightarrow \mathcal{X} \times \mathbf{R}$, where $\mathcal{U} \subset K$ is a neighbourhood of 0 , that satisfies $\Phi(f(k))=0$ for every $k \in \mathcal{U}$. This completes the proof of the Main Theorem.

\section{Deformations of the SCAFFold}

The main theorem answers the question of the existence of minimal Lagrangian submanifolds with boundary on the scaffold $W$ which are near the given candidate $L$. A relatively simple extension of the theory that has been developed so far can be used to answer the question of the existence of minimal Lagrangian submanifolds on neighbouring scaffolds. If $W^{\prime}$ is a symplectic scaffold near $W$ and there is a special Lagrangian submanifold $L$ with boundary $\partial L \subset W$, one asks whether there is a 
special (or minimal) Lagrangian submanifold $L^{\prime}$ near $L$ with boundary $\partial L^{\prime} \subset W^{\prime}$. There is an affirmative answer to this question.

Corollary 9. Let $L$ be a special Lagrangian submanifold of a Calabi-Yau manifold $M$ whose boundary lies on a symplectic, codimension two scaffold $W$. Furthermore, suppose that the topology of $L$ forces its first Betti number $b^{1}(L)$ to vanish. Then if $W^{\prime}$ is any symplectic, codimension two submanifold of $M$ that is sufficiently near $W$ in the sense that $W^{\prime}$ can be written as $\phi_{X}(W)$ for some $X \in C^{1, \beta}\left(\Gamma\left((T W)^{\omega}\right)\right)$ which is sufficiently small, then there is a minimal Lagrangian submanifold $L^{\prime}$ near $L$ and with boundary on $W^{\prime}$.

To prove this result, it is necessary first to parametrize nearby scaffolds over a Banach space in some way. The symplectic structure preserving Hamiltonian deformations of $W$ will be used for this purpose: a procedure will be developed which associates a time-one Hamiltonian flow to each element of the set of $C^{2, \beta}$ sections of the two-dimensional bundle $(T W)^{\omega}$.

Let $X$ be a $C^{2, \beta}$ section in $\Gamma\left((T W)^{\omega}\right)$ and suppose $\mathcal{U}$ is a tubular neighbourhood of $W$ which is symplectomorphic to $W \times \mathbf{R}^{2}$. Furthermore, suppose that the coordinate vector fields $\frac{\partial}{\partial s^{1}}$ and $\frac{\partial}{\partial s^{2}}$ (guaranteed by Lemma 5 ) coincide with the unit normal vector field $N$ and the vector field $J N$, respectively, over the boundary $\partial L \subset W$. Write $X$ in these coordinates as

$$
X(q)=a^{1}(q) \frac{\partial}{\partial s^{1}}+a^{2}(q) \frac{\partial}{\partial s^{2}}
$$

where $q \in W$ and the $a^{i}$ are functions of $W$. Now let $\eta: M \longrightarrow \mathbf{R}$ be a positive, $C^{\infty}$ cut-off function equal to zero outside $\mathcal{U}$ and equal to one inside a smaller tubular neighbourhood of $W$, and define the function $H_{X}: M \longrightarrow \mathbf{R}$ by

$$
H_{X}(q, s)=\eta(q, s)\left(-a^{2}(q) s^{1}+a^{1}(q) s^{2}\right)
$$

for $(q, s) \in \mathcal{U}$ and make $H_{X}$ equal to zero elsewhere. Because the symplectic form of $W \times \mathbf{R}^{2}$ is equal to $\left.\omega\right|_{W}+\mathrm{d} s^{1} \wedge \mathrm{d} s^{2}$, it is easy to see that the Hamiltonian vector field associated to $H_{X}$ is equal to $X$ when $s^{1}=s^{2}=0$; that is, on the submanifold $W$ itself. Finally, let $\phi_{X}: M \longrightarrow M$ denote the time-one Hamiltonian flow associated to the function $H_{X}$. By elementary properties of the flow, it is clear that

$$
\left.\frac{\mathrm{d}}{\mathrm{d} t} \phi_{t X}\right|_{t=0}=J \nabla H_{X}
$$

and if this quantity is restricted to $W$, then it equals $X$.

The map $X_{q} \mapsto \phi_{X}(q)$ for $X_{q} \in\left(T_{q} W\right)^{\omega}$ is a local diffeomorphism because (9) implies that its linearization at the zero section is the identity. Without loss of generality, one can assume that it is the tubular neighbourhood $\mathcal{U}$ that is diffeomorphic to a neighbourhood of the zero section in $\left(T_{q} W\right)^{\omega}$. Hence, any scaffold $W^{\prime}$ sufficiently near $W$ and sufficiently $C^{1}$-regular (to ensure that $W^{\prime}$ projects onto $W)$ is a Hamiltonian deformation of the form $W^{\prime}=\phi_{X}(W)$ for some vector field $X \in \Gamma\left((T W)^{\omega}\right)$ that is sufficiently close to the zero section. The $C^{1, \beta}$ sections of the bundle $(T W)^{\omega}$ can thus be used to parametrize scaffolds sufficiently close to $W$.

This parametrization of nearby scaffolds leads to the following deformation operator. Define the map $\Phi_{1}: C^{1, \beta}\left(\Gamma\left((T W)^{\omega}\right)\right) \times \mathcal{X} \times \mathbf{R} \longrightarrow C^{0, \beta}\left(\mathrm{d} \Lambda^{1}(L)\right) \times$ 
$C^{0, \beta}\left(\mathrm{d} \Lambda^{n-1}(L)\right)$ by

$$
\Phi_{1}(X, V, \theta)=\left(\phi_{X} \circ \widehat{\exp }(V)\right)^{*}\left(\omega,-\mathbf{I m}\left(\mathrm{e}^{-\mathrm{i} \theta} \Omega\right)\right) .
$$

If $\Phi_{1}(X, V, \theta)=(0,0)$, then the submanifold $L^{\prime}=\phi_{X} \circ \widehat{\exp }(V)(L)$ is minimal Lagrangian with calibration form $\operatorname{Re}\left(\mathrm{e}^{-\mathrm{i} \theta} \Omega\right)$. Furthermore, $\partial L^{\prime}$ is contained in $W^{\prime}=\phi_{X}(W)$ because the deformation $\widehat{\exp }(V)$ preserves $W$. The parametrization and deformation operator constructed here now lead to the proof of the corollary.

Proof of Corollary 9 The linearization of $\Phi_{1}$ in the $\mathcal{X} \times \mathbf{R}$ directions remains the operator from equation (31), and is thus an isomorphism because the triviality condition $b^{1}(L)=0$ has been assumed. Therefore, the Implicit Function Theorem implies that there is an open set $\mathcal{U}$ of 0 in $C^{1, \beta}\left(\Gamma\left((T W)^{\omega}\right)\right)$ and a map $G: \mathcal{U} \rightarrow \mathcal{X} \times \mathbf{R}$ satisfying $\Phi_{1}(X, G(X))=(0,0)$. Suppose $G(X)=(V(X), \theta(X))$. Then the submanifold $\phi_{X} \circ \widehat{\exp }(V(X))(L)$ is minimal and Lagrangian, calibrated by the differential form $\operatorname{Re}\left(\mathrm{e}^{-\mathrm{i} \theta(X)} \Omega\right)$ and has boundary on the scaffold $\phi_{X}(W)$ (this is symplectic because $\phi_{X}$ is a symplectomorphism). Consequently, if $W^{\prime}$ is any codimension 2, symplectic submanifold of the form $\phi_{X}(W)$ with $X \in \mathcal{U}$, then the minimal submanifold with boundary on $W^{\prime}$ required to prove the corollary is simply $\phi_{X} \circ \widehat{\exp }(V)(L)$.

\section{ACKNOWLEDGEMENTS}

I thank my Ph.D. advisor at Stanford University, Rick Schoen, for his patience, insight and his confidence in me while I was carrying out the research for this paper. I also thank Justin Corvino and Vin de Silva for their inspirations, ideas, and careful proofreading.

\section{REFERENCES}

1. R. Abraham, J. E. Marsden, and T. Ratiu, Manifolds, Tensor Analysis, and Applications, second ed., Springer-Verlag, New York, 1988. MR 89f:58001

2. Adrian Butscher, Regularizing a singular special Lagrangian variety, Submitted February 2001.

3. Reese Harvey and H. Blaine Lawson, Jr., Calibrated geometries, Acta Math. 148 (1982), 47-157. MR 85i:53058

4. Mark Haskins, Constructing special Lagrangian cones, math.DG/0005164.

5. Nigel J. Hitchin, The moduli space of special Lagrangian submanifolds, Ann. Scuola Norm. Sup. Pisa Cl. Sci. (4) 25 (1997), no. 3-4, 503-515, Dedicated to Ennio De Giorgi. MR 2000c:32075

6. Dominic Joyce, Constructing special Lagrangian $m$-folds in $\mathbf{C}^{m}$ by evolving quadrics, math.DG/0008154. Math. Ann. 320 (2001), 757-797.

7. $\ldots$, Evolution equations for special Lagrangian 3-folds in $\mathbf{C}^{3}$, math.DG/0010036. Ann. Global Anal. and Geom. 20 (2001), 345-403.

8. L Lectures on Calabi-Yau and special Lagrangian geometry, math.DG/0108088.

9. 85 (2002), 233-256.

10. math.DG/0011179.

11. _ Special Lagrangian 3-folds and integrable systems, math.DG/0101249.

12. S S S S Lecial Lagrangian $m$-folds in $\mathbf{C}^{m}$ with symmetries, math.DG/0008021.

13. Dusa McDuff and Dietmar Salamon, Introduction to Symplectic Topology, Second ed., The Clarendon Press Oxford University Press, New York, 1998. MR 2000g:53098

14. Robert C. McLean, Deformations of calibrated submanifolds, Comm. Anal. Geom. 6 (1998), no. 4, 705-747. MR 99j:53083 
15. Sema Salur, Deformations of special Lagrangian submanifolds, Commun. Contemp. Math. 2 (2000), no. 3, 365-372. MR 2002g:53094

16. R. Schoen and J. Wolfson, Minimizing volume among Lagrangian submanifolds, Differential Equations: La Pietra 1996 (Shatah Giaquinta and Varadhan, eds.), Proc. of Symp. in Pure Math., vol. 65, 1999, pp. 181-199. MR 99k:53130

17. Richard Schoen, Lecture Notes in Geometric PDEs on Manifolds, Course given in the Spring of 1998 at Stanford University.

18. Günter Schwarz, Hodge Decomposition-A Method for Solving Boundary Value Problems, Springer-Verlag, Berlin, 1995. MR 96k:58222

Max Planck Institute for Gravitational Physics, am Muehlenberg 1, 14476 Golm Brandenburg, Germany

E-mail address: butscher@aei-potsdam.mpg.de

Current address: Department of Mathematics, University of Toronto at Scarborough, Scarborough, Ontario, Canada M1C 1A4

E-mail address: butscher@utsc.utoronto.ca 